\title{
Experimental determination of translational start sites resolves uncertainties in genomic open reading frame predictions - application to Mycobacterium tuberculosis
}

\author{
Katherine L. Smollett,† Amanda S. Fivian-Hughes,† Joanne E. Smith, \\ Anchi Chang, Tara Raoł and Elaine O. Davis \\ Division of Mycobacterial Research, MRC National Institute for Medical Research, The Ridgeway, \\ Mill Hill, London NW7 1AA, UK
}

Correspondence

Elaine O. Davis

edavis@nimr.mrc.ac.uk

Received 1 August 2008

Revised 11 September 2008

Accepted 25 September 2008

\begin{abstract}
Correct identification of translational start sites is important for understanding protein function and transcriptional regulation. The annotated translational start sites contained in genome databases are often predicted using bioinformatics and are rarely verified experimentally, and so are not all accurate. Therefore, we devised a simple approach for determining translational start sites using a combination of epitope tagging and frameshift mutagenesis. This assay was used to determine the start sites of three Mycobacterium tuberculosis proteins: LexA, SigC and Rv1955. We were able to show that proteins may begin before or after the predicted site. We also found that a small, non-annotated open reading frame upstream of Rv1955 was expressed as a protein, which we have designated Rv1954A. This approach is readily applicable to any bacterial species for which plasmid transformation can be achieved.
\end{abstract}

\section{INTRODUCTION}

Mapping the protein translational start site for genes is essential to define the protein sequence, intergenic distances and upstream DNA regions which may contain regulatory motifs. Correct identification of intergenic distances is important for predicting operons and small untranslated RNAs (Carter et al., 2001; Moreno-Hagelsieb \& Collado-Vides, 2002). Therefore correct identification of translational start sites is necessary for understanding both protein function and transcriptional regulation (Makita et al., 2007; Rison et al., 2007).

There are many programs for determining open reading frames (ORFs) across genomes using mathematical, probabilistic models. One of the most widely used for prokaryotic genomes is GLIMMER, which tends to use the first possible translation initiation codon (ATG, GTG or TTG) for a particular gene, giving the longest possible ORF (Delcher et al., 1999). Other approaches take into account factors such as the location of ribosome-binding sites and protein sequence comparisons (Besemer et al., 2001; Makita et al., 2007; Nielsen \& Krogh, 2005). However,

†These authors contributed equally to this work.

¥Present address: School of Biosciences, The University of Birmingham, Edgbaston, Birmingham B15 2TT, UK.

Abbreviations: ACDP, Advisory Committee on Dangerous Pathogens; RACE, rapid amplification of cDNA ends; SDM, site-directed mutagenesis. due to the larger amount of validated data, these methods are often optimized for Escherichia coli and the accuracy of these predictions, particularly with regard to the translational start site, can vary for different genomes (Makita et al., 2007). The inaccuracy of ORF and translational start site predictions is particularly a problem with GC-rich genomes, such as that of Mycobacterium tuberculosis, due to the more frequent occurrence of the GTG start codon, which is often more common than stop codons (TAA, TGA, TAG), resulting in more possible start codons for each stop codon than in AT-rich organisms (Nielsen \& Krogh, 2005). Therefore, the annotated start codons for genes are not necessarily correct, and where more than one possible start codon occurs it is important to verify these experimentally.

Experimental methods for determining translational start sites of proteins are cumbersome. Edman degradation is a commonly used approach. In this method the amino acids of a protein or peptide are removed one by one to determine its sequence (Edman, 1950). More recently an experimental approach based on mass spectrometry was developed and applied to M. tuberculosis (Rison et al., 2007). In this method cellular proteins are separated by $2 \mathrm{D}$ electrophoresis, individual protein spots are trypsindigested, and the peptide masses are determined by mass spectrometry. The translational start site is then determined by comparing the actual mass of the $\mathrm{N}$-terminal peptide to those generated in silico for alternative start sites. 
This technique can be used as a high-throughput method to assess the translational start sites of many proteins as part of the determination of the bacterial proteome. However, it is likely that many proteins will be missed by this technique as many proteins do not form visible spots on $2 \mathrm{D}$ gels and the $\mathrm{N}$-terminal peptides generated may not be detected by mass spectrometry.

Therefore, we devised a simple approach for determining translational start sites of individual proteins of interest using a combination of epitope tagging and frameshift mutagenesis. It has previously been demonstrated that the C-terminal portion of the product of the human protooncogene $c-m y c$ can be used as an epitope tag for the detection of recombinant proteins in mycobacteria (Spratt et al., 2005). In this assay for determining protein translational start sites, the genes of interest along with their promoter regions were cloned into a vector to give an in-frame C-terminal $m y c$ tag sequence and single-residue deletions were created between potential start codons, avoiding any potential promoter regions or ribosomebinding sites. Therefore, if the deletion occurs downstream of the actual translational start codon then the resulting protein, including the tag, would be out of frame. Deletions upstream of the start codon will not affect the frame of the protein. Whether the protein is in-frame or out of frame can be detected by the presence or absence of the Myctagged protein in cell-free extract. This assay was used to determine the start sites of the M. tuberculosis proteins LexA, SigC and Rv1955. We demonstrated that proteins may begin before or after the predicted site. We were also able to use this technique to show that a small, nonannotated ORF was expressed as a protein.

\section{METHODS}

Bacterial strains and media. Escherichia coli strain $\mathrm{DH} 5 \alpha$ (Invitrogen) was used for all plasmid construction and strain XL1Blue (Stratagene) was used for site-directed mutagenesis (Sambrook et al., 1989). The mycobacterial strains used were Mycobacterium smegmatis mc $^{2} 155$ (Snapper et al., 1990) and M. tuberculosis H37Rv. E. coli was grown in Luria-Bertani (LB) broth or on LB agar plates, while $M$. smegmatis and $M$. tuberculosis were grown in modified Dubos medium (Difco) supplemented with $4 \%$ albumin and $0.2 \%$ (w/v) glycerol or on Difco Middlebrook 7H11 agar (Beckton Dickinson) plates supplemented with $4 \%$ albumin and $0.5 \%(\mathrm{w} / \mathrm{v})$ glycerol. E. coli and M. smegmatis liquid cultures were grown at $37{ }^{\circ} \mathrm{C}$ with shaking at 225 r.p.m. and M. tuberculosis was grown at $37{ }^{\circ} \mathrm{C}$ in a rolling incubator at 2 r.p.m. All procedures with live $M$. tuberculosis were carried under ACDP containment level 3 conditions. Where appropriate $50 \mu \mathrm{g}$ kanamycin $\mathrm{ml}^{-1}$ (for E. coli) or $25 \mu \mathrm{g}$ kanamycin $\mathrm{ml}^{-1}$ (for mycobacteria) was added to the media.

Plasmid construction. The plasmids used and their construction are described in Table 1, and the primers used in this study are listed in Table 2. All site-directed mutagenesis was performed using the Quickchange site-directed mutagenesis (SDM) kit (Stratagene). All constructs were verified by DNA sequencing.

Preparation of cell-free extracts and Western blotting. Mycobacterial cultures were grown to mid-exponential phase
$\left(\mathrm{OD}_{600}\right.$ 0.6) unless otherwise stated; the bacteria were harvested, washed three times in PBS and cell-free extract prepared as described previously (Davis et al., 2002). In the case of M. tuberculosis extracts, the supernatant was filtered through a low-binding Durapore $0.22 \mu \mathrm{m}$ membrane filter (Ultrafree-MC; Millipore) to ensure complete removal of bacteria before removal from containment facilities. Where mitomycin $\mathrm{C}$ induction was required, mycobacterial cultures were grown to early exponential phase $\left(\mathrm{OD}_{600} 0.3\right)$ and were then split into two; one culture was induced with $0.02 \mu \mathrm{g}$ mitomycin $\mathrm{C} \mathrm{ml}^{-1}$ and the other was an uninduced control. Both were then incubated at $37{ }^{\circ} \mathrm{C}$ for $24 \mathrm{~h}$ before harvesting and preparing cell-free extract as above.

Cell-free extracts were separated by SDS-PAGE and the proteins were electroblotted onto a PVDF membrane using a semi-dry blotter (Hybaid) at $1 \mathrm{~mA} \mathrm{~cm}^{-2}$ for $1 \mathrm{~h}$. Equal loading of the cell-free extract was confirmed by Coomassie blue staining of an identical gel. Western blotting was performed using rabbit anti-Myc (A-14, Santa Cruz) as the primary antibody, at a 1:2000 dilution, and anti-rabbit conjugated to horseradish peroxidase (Dako) as the secondary antibody, at a 1:5000 dilution. The blot was developed using ECL Western blotting detection reagents (GE Healthcare) according to the manufacturer's instructions.

Preparation of RNA and RT-PCR. RNA was prepared from mycobacteria using the FastRNA Pro Blue kit (Qbiogene). Contaminating DNA was removed using the TURBO DNA-free kit (Ambion) and first-strand DNA synthesis was performed using Superscript II (Invitrogen) with random primers (Promega). To confirm the presence of the specific transcript, PCR was then performed on the cDNA using the reverse primer MycRTR with forward primers LexARTF, SigCRTF, JORTF or Rv1955RTF (Table 2) as appropriate. To assess whether ORF2 is co-transcribed with Rv1955-Rv1957 within M. tuberculosis H37Rv, PCR was performed on CDNA using forward primer ORF2F with reverse primers ORF2Rv1955R, ORF2-Rv1956R or ORF2-Rv1957R (Table 2) as appropriate. PCR was also performed on RNA without reverse transcriptase to control for DNA contamination.

5' RACE transcriptional start site mapping. 5' RACE System for Rapid Amplification of cDNA Ends (Version 2.0; Invitrogen) was performed to map the transcriptional start site of Rv1955 according to the manufacturer's guidelines, using primers Rv1955 GSP1, GSP2 and GSP3. cDNA was tailed at the $3^{\prime}$ ends with poly-cytosine and transcriptional start sites were identified at the junction with the polycytosine tail.

Sequence analysis. DNA analysis and potential ORF identification was performed using SeqBuilder (DNASTAR) and Translator (http:// www.fr33.net/translator). Sequence analysis was performed using CLUSTAL W (http://www.ebi.ac.uk/Tools/clustalw2/index.html) and BLAST (http://www.ncbi.nlm.nih.gov/blast/Blast.cgi).

\section{RESULTS}

\section{Determination of the translational start site of LexA}

The translational start site for LexA was initially assigned by aligning the predicted $M$. tuberculosis LexA sequence with other available LexA homologues, primarily those of E. coli and Bacillus subtilis (Movahedzadeh et al., 1997). However, more recently, the sequences of LexA from other mycobacterial species have become available (Brosch et al., 2007; Cole et al., 2001; Stinear et al., 2007). Alignment of 
Table 1. Plasmids used in this study

\begin{tabular}{|c|c|c|}
\hline Name & Description and construction* & Reference \\
\hline pMV306 & Integrating mycobacterial cloning vector & Stover et al. (1991) \\
\hline pEJ410 & Deletion of part of polylinker between $X b a \mathrm{I}$ and $M l u \mathrm{I}$ in pMV306 & This study \\
\hline pEJMyc & $\begin{array}{l}\text { Myc tag vector; insertion of annealed oligonucleotides mycF and mycR into pEJ410 } \\
\text { between EcoRI and EcoRV }\end{array}$ & This study \\
\hline pKS03 & $\begin{array}{l}\text { pEJMyc containing SigC and } 644 \text { bp upstream of H37Rv annotated start; insertion of } \\
1199 \text { bp PCR product from primers SigCupclone and SigCdownclone into HindIII and EcoRV }\end{array}$ & This study \\
\hline pKS03mut2 & pKS03 derivative missing residue $2 \dagger$; SDM using primers SigCmut $2 \mathrm{~F}$ and SigCmut $2 \mathrm{R}$ & This study \\
\hline pKS03mut3 & pKS03 derivative missing residue $3 \dagger ;$ SDM using primers SigCmut $3 \mathrm{~F}$ and SigCmut3R & This study \\
\hline pKS03mut4 & pKS03 derivative missing residue $4 \dagger ;$ SDM using primers SigCmut4F and SigCmut4R & This study \\
\hline pKS03mut5 & pKS03 derivative missing residue $5 \dagger ;$ SDM using primers SigCmut5F and SigCmut5R & This study \\
\hline pKS03mut6 & pKS03 derivative missing residue $6 \dagger$; SDM using primers SigCmut6F and SigCmut6R & This study \\
\hline pJS014 & $\begin{array}{l}\text { pEJMyc containing } 654 \text { bp region upstream of H37Rv SigC annotated start site; insertion of } \\
654 \text { bp PCR product from primers SigCupclone and RSigC into HindIII and XbaI }\end{array}$ & This study \\
\hline pKS04 & $\begin{array}{l}\text { pEJMyc containing LexA and } 318 \text { bp upstream of annotated start; insertion of } 968 \text { bp PCR } \\
\text { product from primers LexAF and LexAR into HindIII and EcoRI }\end{array}$ & This study \\
\hline pKS04mut1 & pKS04 derivative missing residue $1 \dagger$; SDM using primers LexAmut1F and LexAmut1R & This study \\
\hline pASF29 & $\begin{array}{l}\text { pEJmyc containing Rv1955 and } 490 \text { bp upstream of annotated start; insertion of } 1006 \text { bp PCR } \\
\text { product from primers Rv1955mycF and Rv1955mycR into PvuII and XbaI }\end{array}$ & This study \\
\hline pASF34 & pASF29 derivative missing residue $1 \dagger$; SDM using primers mycFS1F and mycFS1R & This study \\
\hline pASF35 & pASF29 derivative missing residue $2 \dagger ;$ SDM using primers mycFS2F and mycFS2R & This study \\
\hline pASF36 & pASF29 derivative missing residue $3 \uparrow$; SDM using primers mycFS3F and mycFS3R & This study \\
\hline pASF37 & $\begin{array}{l}\text { pASF29 derivative where ORF1 is in-frame with C-terminal Myc tag; SDM using primers } \\
\text { mycORF1F and mycORF1R }\end{array}$ & This study \\
\hline pASF38 & $\begin{array}{l}\text { pASF29 derivative where ORF2 is in-frame with C-terminal Myc tag; SDM using primers } \\
\text { mycORF2F and mycORF2R }\end{array}$ & This study \\
\hline
\end{tabular}

${ }^{\star}$ SDM, site-directed mutagenesis.

$\dagger$ For location of missing residues see Fig. 2(a), 3(a) or 4(a).

the various predicted mycobacterial LexA protein sequences, including those of $M$. avium, M. leprae, $M$. ulcerans, M. smegmatis and M. bovis (Fig. 1), revealed that all of the mycobacterial LexA sequences examined, with the exception of the $M$. bovis protein, included 15-26 extra amino acids at the $\mathrm{N}$ terminus compared to $M$. tuberculosis LexA as predicted from the annotated translational start site. The additional amino acid sequence resulting from translation of $M$. tuberculosis LexA from an upstream alternative start site showed a strong similarity with the amino acid sequences of the $\mathrm{N}$-terminal region of the other mycobacterial LexAs, indicating the possibility that the $M$. tuberculosis LexA is longer than previously thought. Therefore we decided to ascertain the M. tuberculosis LexA translational start site experimentally.

The lexA gene, including its promoter region, was cloned into $\mathrm{pEJMyc}$ to give $\mathrm{pKS} 04$, giving LexA with an in-frame C-terminal Myc tag. LexA contains two possible start codons (Fig. 2a): the annotated start site and a potential alternative start site 57 bases upstream coincident with the transcriptional start site. Therefore, one residue was deleted between these possible start codons to give pKS04mut1 (Fig. 2a). The plasmids pKS04, pKS04mut1 and the pEJMyc vector were then transformed separately into $M$. smegmatis strain $\mathrm{mc}^{2} 155$, a commonly used model strain for M. tuberculosis. Cell-free extract was prepared from each strain and the presence or absence of Myc-tagged protein was determined by Western blotting (Fig. 2b). A $27.6 \mathrm{kDa}$ band corresponding to Myc-tagged LexA was detected in cell-free extract from M. smegmatis containing pKS04 but not in cell-free extract from M. smegmatis containing pKS04mutl or pEJMyc. We tested for the presence of a specific mRNA transcript of myc-tagged lexA, to determine that the inability to detect Myc-tagged protein in these strains was due to lack of translation of the tag and not a lack of transcription. RT-PCR was performed using the primers LexARTF and MycRTF, which amplify 195 nucleotides at the $3^{\prime}$ end of the transcript, including the $m y c$ sequence. The presence of lexA-myc transcript was detected in RNA isolated from $M$. smegmatis containing pKS04 and pKS04mut1 but not $\mathrm{pEJMyc}$ or the reverse-transcriptase-negative controls (Fig. 2c). pEJMyc, pKS04 and pKS04mutl were then transformed into M. tuberculosis strain $\mathrm{H} 37 \mathrm{Rv}$ to confirm that the translation start site is the same in both mycobacterial species. The presence of LexA-Myc was detected in cell-free extract from $M$. tuberculosis expressing $\mathrm{pKS} 04$ but not pKS04mut1 or pEJMyc (Fig. 2d). The presence of RNA transcript for lexA-myc was confirmed by RT-PCR as above (Fig. 2e). Therefore the translational start site for LexA is upstream of residue 1, indicating that LexA is not 
Table 2. Primers used in this study

\begin{tabular}{|c|c|}
\hline Name & Sequence $5^{\prime}-3^{\prime}$ \\
\hline $\mathrm{MycF}$ & AATTCGCGGCCGATATCTAGAACAAAAACTCATCTCAGAAGAG \\
\hline MycR & CAGATCCTCTTCTGAGATGAGTTTTTGTTCTAGATATCGGCCGCG \\
\hline LexAF & AGAAAGCTTCCGGTGTCATGTTCGCTCCT \\
\hline LexAR & AGAGAATTCATGACCTTGCGGATCACCGTGAC \\
\hline LexAmut1F & CGACTACATTCATTGCCATGAAGACAGCAACGACACCTCGGTTGC \\
\hline LexAmut1R & GCAACCGAGGTGTCGTTGCTGTCGTTCATGGCAATGAATGTAGTCG \\
\hline SigCupclone & GCTGCGGAGGAAGCTTGTAAATGCCGCGGTG \\
\hline SigCdownclone & ATCCGCCGGTGAGGTCGTCGGGCTCCGCGTC \\
\hline RSigC & TCTCTAGAGCGGGCATAAACCATAGCCCGGCAGG \\
\hline SigCmut2F & CGCTCCGGCCGGCAAGGTTGCGATGCCGGACGGGCCCCGC \\
\hline SigCmut2R & GCGGGGCCCGTCCGGCATCGCAACCTTGCCGGCCGGAGCG \\
\hline SigCmut3F & GCCGTAGTATCACACGCGCGAGATGGCAGGCGCCAAAGCG \\
\hline SigCmut3R & CGCTTTGGCGCCTGCCATCTCGCGCGTGTGATACTACGGC \\
\hline SigCmut4F & GTATCACACGCGCGCAGATGCAGGCGCCAAAGCGCATTCG \\
\hline SigCmut4R & CGAATGCGCTTTGGCGCCTGCATCTGCGCGCGTGTGATAC \\
\hline SigCmut5F & GGCTATGGTTTATGCCCATGCCGCGACGGCAAGCGACGAC \\
\hline SigCmut5R & GTCGTCGCTTGCCGTCGCGGCATGGGCATAAACCATAGCC \\
\hline SigCmut6F & GGCTATGGTTTATGCCATGACCGCGACGGC \\
\hline SigCmut6R & GCCGTCGCGGTCATGGCATAAACCATAGCC \\
\hline Rv1955 GSP1 & ATCGCAACTCCAGAATGTC \\
\hline Rv1955 GSP2 & AATACCGGCCGGCCATCCACAG \\
\hline Rv1955 GSP3 & TGGATCAGGGGGCGGCACAG \\
\hline Rv1955mycF & GTACAGCTGCGCACCGACCCGACCCAGA \\
\hline Rv1955mycR & AGCTCTAGACCGATCGGTGGGGTGTCGCC \\
\hline mycFS1F & CGCGGCTGAGCTTGTGCCGTGGGATGGGTGTCGCACCGTC \\
\hline mycFS1R & GACGGTGCGACACCCATCCCACGGCACAAGCTCAGCCGCG \\
\hline mycFS2F & GACAAGACCAACTGTGCCGCCCCTGATCCAGCCGCCATGG \\
\hline mycFS2R & CCATGGCGGCTGGATCAGGGGCGGCACAGTTGGTCTTGTC \\
\hline mycFS3F & GATCCAGCCGCCATGGGTACTGGAAGTTCTTCCGGGCATC \\
\hline mycFS3R & GATGCCCGGAAGAACTTCCAGTACCCATGGCGGCTGGATC \\
\hline mycORF1F & GGTTGGCGGTGGCAACGAGGGAACAAAAACTCATCTCAG \\
\hline mycORF1R & CTGAGATGAGTTTTTGTTCCCTCGTTGCCACCGCCAACC \\
\hline mycORF2F & GAACAAGAGCGGCCGCGGCGAACAAAAACTCATCTCAG \\
\hline mycORF2R & CTGAGATGAGTTTTTGTTCGCCGCGGCCGCTCTTGTTC \\
\hline MycRTR & CCTCTTCTGAGATGAGTTTTTG \\
\hline SigCRTF & TCGAGGTAACCACGATGATC \\
\hline LexARTF & CCACCGTCAAGACGTTCAAA \\
\hline Rv1955RTF & AGTTCTTCCGGGCATCTGT \\
\hline JORTF & TTGCGCATGCCGTAGTATCA \\
\hline ORF2F & TGCCGGTGCTGGTGGTCATC \\
\hline ORF2-Rv1955R & GTCGCCGAAGGCTCTTTTCCAG \\
\hline ORF2-Rv1956R & TGGGGTCGCTGGGTTCCTTCT \\
\hline ORF2-Rv1957R & GGCGGCGTATGCCGTAAGTTCT \\
\hline
\end{tabular}

translated from the annotated start site and is instead translated from a start codon $57 \mathrm{bp}$ further upstream at the transcriptional start site.

\section{Determination of the translational start site of SigC}

The predicted translational start site for the sigma factor SigC in M. tuberculosis differs in the annotation for strain H37Rv compared to that for strain CDC1551 (Camus et al., 2002; Fleischmann et al., 2002). Although the two nucleotide sequences are identical, the annotated translational start of sigC for strain CDC1551 is $378 \mathrm{bp}$ upstream of that of strain $\mathrm{H} 37 \mathrm{Rv}$, giving an extra 126 aa at the $\mathrm{N}$ terminus. Starting at this start site would also result in a 244 bp overlap with blaC, encoded on the opposite strand. Therefore, we decided to determine which of these translational start sites was correct.

The sigC gene and its promoter region was cloned into pEJMyc to give $\mathrm{pKS} 03$, containing sigC with an in-frame Cterminal Myc tag. There are five alternative start codons upstream of the annotated sigC translational start site. 


M. tuberculosis
M. avium
M. leprae
M. ulcerans
M. smegmatis
M. bovis
B. subtilis
E. coli
M. tuberculosis
M. avium
M. leprae
M. ulcerans
M. smegmatis
M. bovis
B. subtilis
E. coli
M. tuberculosis
M. avium
M. leprae
M. ulcerans
M. smegmatis
M. bovis
B. subtilis
E. coli
M. tuberculosis
M. avium
M. leprae
M. ulcerans
M. smegmatis
M. bovis
B. subtilis
E. coli
M. tuberculosis
M. avium
M. leprae
M. ulcerans
M. smegmatis
M. bovis
B. subtilis
E. coli
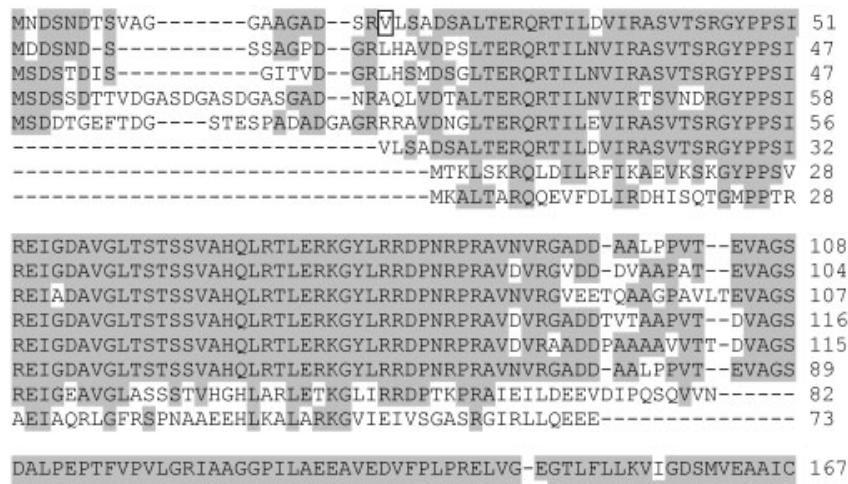
DALPEPTFVPVLGRIAAGGPILAEEAVEDVFPLPRELVG-DGTLFLLKVVGDSMVEAAIC 163 DVLPEPTFVP I LGRIAAGSPI FAEGTVEDI FPLPRELVG-EGTLFLLKVTGDSMVEAAIC 166 DALPEPTEVPVLGRIAAGGPILAEEAVEDVFPLPRELVG-QGTLFLLKVVGESMVEAAIC 175 DALPEPTEVPVLGRIAAGGPILAEEAVEDVFPLPRELVG-EGSLFLLKVVGDSMVDAAIC 174 DALPEPTFAPVLGRIAAGGPILAEEAVEDVFPLPRELVG-EGTLFLLKVIGDSMVEAAIC 148 $\begin{array}{lll}\text { DALPEPTEAPVLGRIAAGGPILAEEAVEDVFPLPRELVG-EGTLFLLKVIGDSMVEAAIC } & 148 \\ --1 & \end{array}$

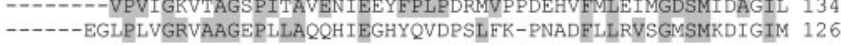

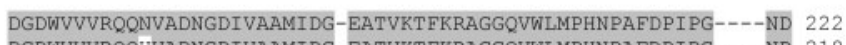
DGDWVVVROUVADNGDIVAAMTDG-EATVKT KRAGGQVWIMPHNPAFPIPG-DGDWVVVRQQKVADNGDIVAAMIDG-EATVKTFKRAGGQVWLIPHNPAFDPIPG----ND 221 DGDWVVVRQQNVADNGDIVAAMIDG-EATVKTFKRAGGQIWLMPHNPAFDPIPG----ND 230 DGDWVVVRQQNVADNGDIVAAMI DG-EATVKTFKRARGQVWLMPHNPAYDPIPG----NE 229 DGDWVVVRQQNVADNGDIVAAMIDG-EATVKTFKRAGGQVWLMPHNPAFDPIPG----ND 203 DKDYVIVKQQNTANNGEIVVAMTEDDEATVKRFYKEDTHIRLQPENPTMEPI IL-----QN 190 DGDLLAVHKTQDVRNGQVVVARIDD-EVTVKRLKKOGNKVELLPENSEFKPIVVDLRQQS 185

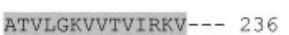

$\begin{array}{ll}\text { ATVLGKVVTVIRKV--- } & 236 \\ \text { ATVLGKVVTVIRKV--- } & 232\end{array}$

ATVLGKVVTVIRKI--- 235

ATVLGKVVTVIRKI--- 244

AAVLGKVVTVIRKI--- 243

ATVLGKVVTVIRKV--- 217

ATVLGKVVTVIRKV--- 217

FTIEGLAVGVIRNGDWL 202
Fig. 1. Comparison of M. tuberculosis LexA with that of other mycobacterial species. $M$. tuberculosis strain H37Rv LexA (accession no. CAB09461) including 19 aa upstream of the annotated start site was compared to LexA from M. avium strain 104 (ABK66179), $M$. leprae strain TN (CAC31384), M. ulcerans strain Agy99 (ABL05545), M smegmatis strain $\mathrm{mc}^{2} 155$ (ABK70187) and $M$ bovis BCG Pasteur strain 1173P2 (CAL72721) as well as LexA from $B$. subtilis (AAA22573) and $E$. coli (AAA24067) using CLUSTAL W. Conserved residues are shaded; the amino acid corresponding to the annotated translational start site of M. tuberculosis LexA is boxed.
Single base pair deletions were created between potential start codons to give plasmids pKS03mut2, pKS03mut3, pKS03mut4, pKS03mut5 and pKS03mut6, where 2-6 refers to the residue deleted (Fig. 3a). pKS03, and its derivatives, were transformed into $M$. smegmatis $\mathrm{mc}^{2} 155$. Cell-free extract was prepared from each strain and the presence or absence of Myc-tagged protein was determined by Western blotting (Fig. 3b). Due to the large number of amino acids between potential start codons, SigC-Myc was expected to be detected between $35.4 \mathrm{kDa}$ and $21.6 \mathrm{kDa}$, for the largest and smallest potential ORFs respectively. Myc-tagged SigC was detected in extract from $M$. smegmatis containing pKS03, pKS03mut2, pKS03mut3 pKS03mut4 and pKS03mut5 at $21.6 \mathrm{kDa}$, but was not detected in extract from M. smegmatis containing pKS03mut6 or pEJMyc. The presence of mRNA transcript for sigC-myc in M. smegmatis carrying pKS03 and pKS03mut6 was confirmed by RTPCR using primers SigCRTF and MycRTR, indicating that the mutation introduced had no effect on transcription (Fig. 3c). pKS03, pKS03mut5 and pKS03mut6 were then transformed into M. tuberculosis H37Rv. SigC-Myc was detected from the cell-free extracts of $M$. tuberculosis bearing pKS03 and pKS03mut5 but not pKS03mut6 or pEJMyc (Fig. 3d). The presence of sigC-myc transcript in $M$. tuberculosis expressing pKS03mut6 was confirmed as above (Fig. 3e). This indicates that translation initiates at the same site in $M$. tuberculosis as in M. smegmatis. Therefore, the translational start site for $\mathrm{SigC}$ is found between residues 5 and 6 , suggesting that the start site annotated for $\mathrm{H} 37 \mathrm{Rv}$ is the correct one for this gene.

This finding contradicts a recent report that the longer, CDC1551 annotation was correct for H37Rv SigC (Thakur et al., 2007). As the experiments to determine the SigC translational start site presented above were all performed on mycobacterial strains grown to mid-exponential phase, and the experiments performed by Thakur et al. (2007) were performed using cell extracts from stationary-phase bacteria, expression of Myc-tagged SigC was compared at different growth phases, to investigate the possibility that translation of SigC initiates at different sites under different conditions. As well as comparing the size of the tagged protein produced by pKS03, pJS014 was constructed, containing the Myc tag fused in-frame to the codon immediately upstream of the start site identified above. $M$. smegmatis $\mathrm{mc}^{2} 155$ or $M$. tuberculosis $\mathrm{H} 37 \mathrm{Rv}$ containing pEJMyc, pKS03 or pJS014 were grown to either midexponential $\left(\mathrm{OD}_{600} \sim 0.6\right)$, late-exponential/early stationary $\left(\mathrm{OD}_{600} \sim 1.0\right)$ or stationary $\left(\mathrm{OD}_{600} \sim 2.0\right)$ phase and cellfree extract was prepared. No SigC-Myc was detected from M. smegmatis or M. tuberculosis bearing either pEJMyc or pJS014 under any of these conditions, and the SigC-Myc from M. smegmatis or M. tuberculosis expressing pKS03 was consistently $21.6 \mathrm{kDa}$ (Fig. 3f). The presence of sigC-myc transcript in strains expressing pJS014 was verified by RTPCR using the primers JORTF and MycRTR. Therefore, we 
(a)

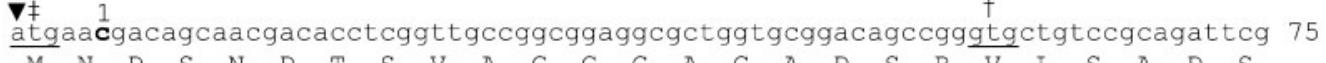

(b)

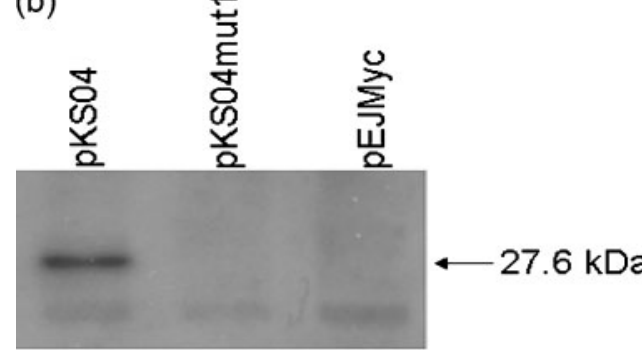

(d)

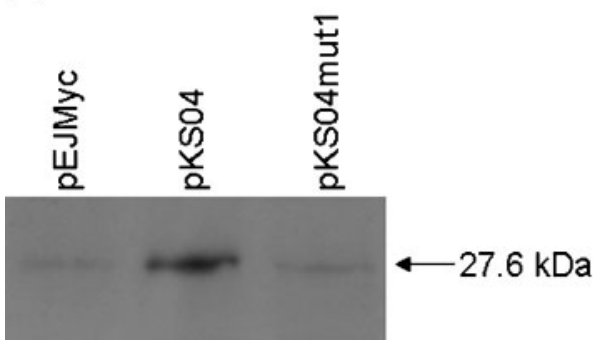

(c)

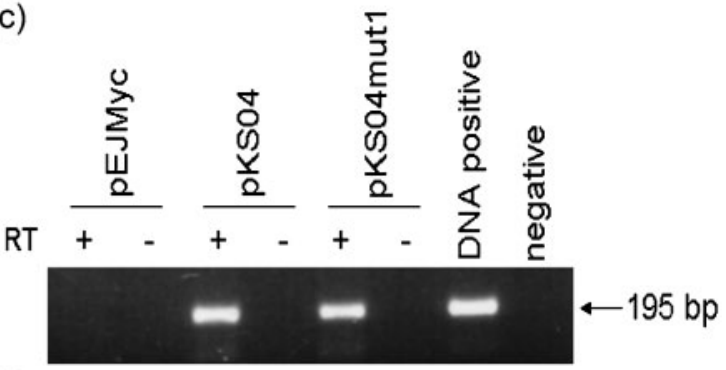

(e)

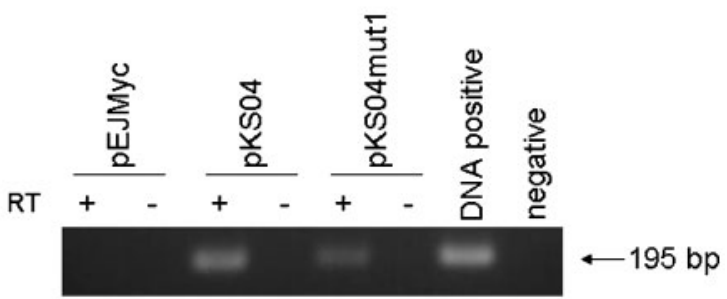

Fig. 2. Identification of the LexA translation start site. (a) The nucleotide sequence, and corresponding amino acid translation, of the beginning of the lexA gene including $57 \mathrm{bp}$ upstream of the annotated start codon. Two possible start codons are underlined; the annotated start site is indicated by $\dagger$, the experimentally determined start codon by $\ddagger$ and the transcriptional start site by $\boldsymbol{\nabla}$. To determine the translational start codon, a single-residue deletion (bold and numbered) was created between the two possible start codons. (b) Western blot analysis of cell-free extracts of $M$. smegmatis containing pEJMyc, pKS04 or pKS04mut1. A $27.6 \mathrm{kDa}$ band corresponding to LexA-Myc can be detected from strains containing pKS04 but not pEJMyc or pKS04mut1. (c) RT-PCR analysis of RNA extracts of M. smegmatis containing pEJMyc, pKS04 or pKS04mut1. A 195 bp band indicating the presence of lexA-myc transcript was detected from strains containing pKS04 and pKS04mut1 but not strains containing pEJMyc or the RNA without reverse transcriptase (RT) controls. (d) Western blot analysis of cell-free extracts of $M$. tuberculosis containing pEJMyc, pKS04 or pKS04mut1. (e) RT-PCR analysis of RNA extracts of M. tuberculosis containing pEJMyc, pKSO4 or pKS04mut1.

have been unable to detect translation of SigC from a site other than the H37Rv annotated start site.

\section{Determination of the transcriptional and translational start sites of Rv1955}

The function of the M. tuberculosis $\mathrm{H} 37 \mathrm{Rv}$ protein Rv1955 is unknown but the gene is thought to be co-transcribed with its two downstream genes, Rv1956, encoding a possible transcriptional regulatory protein, and Rv1957, encoding another protein of unknown function (Cole et al., 1998; Rand, 2003). A promoter motif, thought to regulate a LexA/RecA-independent DNA damage response mechanism in $M$. tuberculosis (designated RecA-NDp, for RecA non-dependent promoter), has been identified approximately $70 \mathrm{bp}$ downstream of the Rv1955 annotated translational start site, suggesting that the predicted annotation is incorrect (Gamulin et al., 2004). Transcriptional start site mapping using $5^{\prime}$ RACE identified two sites for Rv1955, at either 85 or $86 \mathrm{bp}$ downstream and at $336 \mathrm{bp}$ upstream of the annotated translation start site, suggesting that two promoters (designated $\mathrm{P} 1$ and $\mathrm{P} 2$ respectively) control the expression of the Rv1955-Rv1957 operon (Fig. 4a). As RACE uses a poly-cytosine tail and the complementing strand was sequenced, sites cannot be precisely mapped where transcription may begin at a guanine residue; hence the approximate location of the $\mathrm{P} 1$ promoter transcriptional start site. The position of the RecA-NDp motif corresponds with this shorter transcript, further supporting implications that this protein may be smaller than expected. We therefore decided to confirm the M. tuberculosis Rv1955 translational start site experimentally.

The Rv1955 gene and its upstream region, including both promoters, was cloned into pEJMyc to give pASF29, containing Rv1955 with an in-frame C-terminal Myc tag. Rv1955 contains three alternative start codons downstream of the P1 promoter and annotated start site. Single base pair deletions were created downstream of the annotated start site and each potential start codon subsequent to P1 within pASF29 to give plasmids pASF34, pASF35 and 
(a)

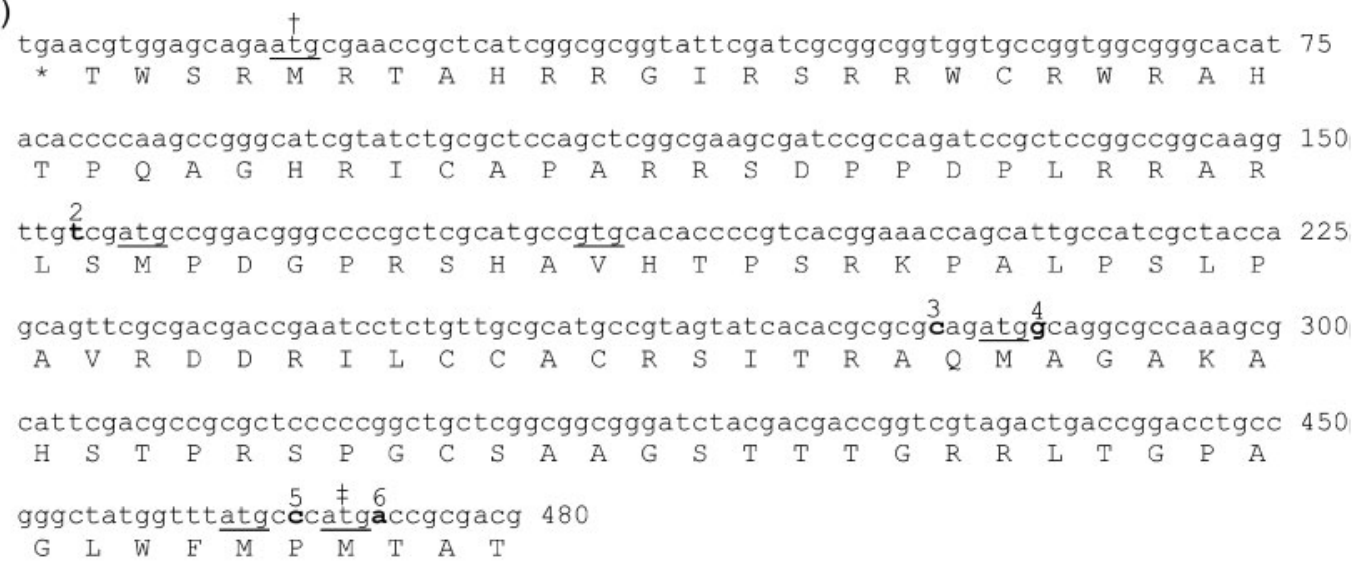
tgaacgtggagcagaatgcgaaccgctcatcggcgcggtattcgatcgcggcggtggtgccggtggcgggcacat 75

a)

(b)
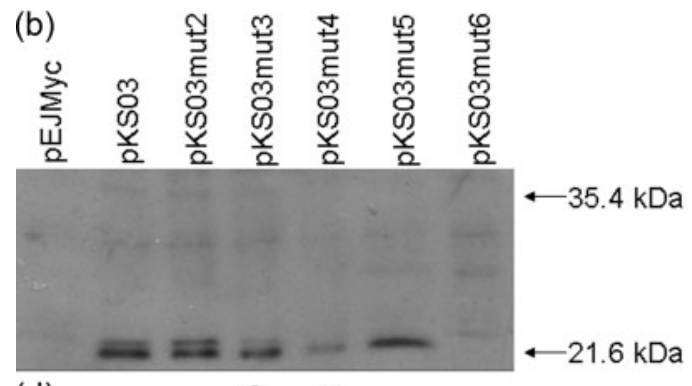

(d)

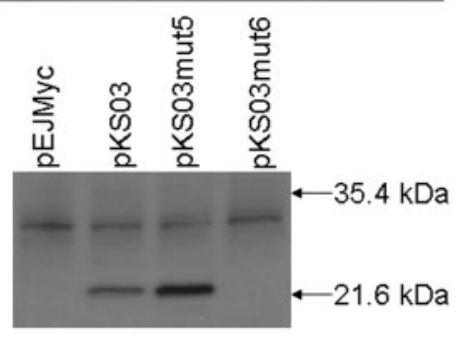

(f)
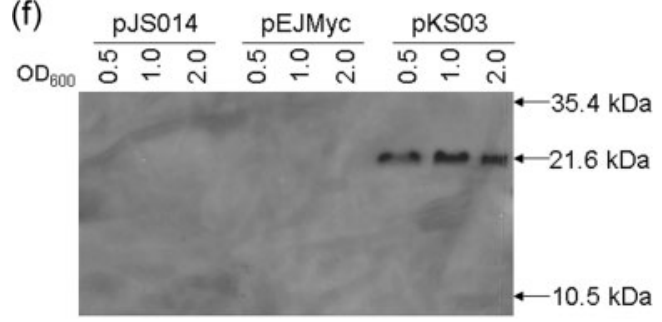

(c)

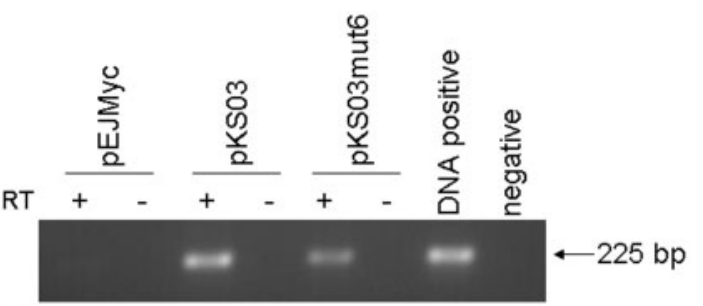

(e)

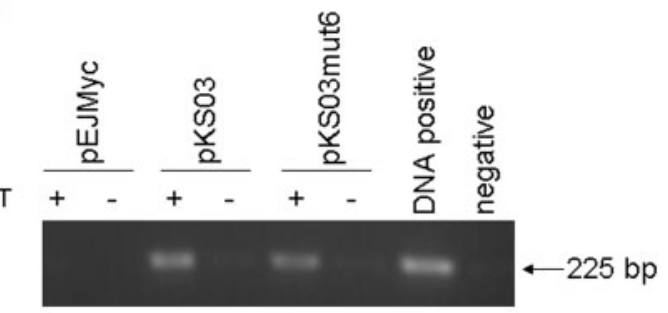

(g)

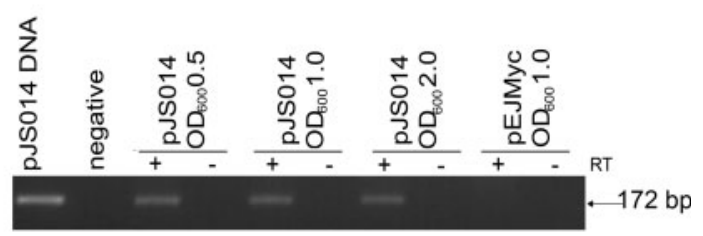

Fig. 3. Identification of the $\mathrm{SigC}$ translational start site. (a) The nucleotide sequence, and corresponding amino acid translation, of the beginning of the sigC gene including $393 \mathrm{bp}$ upstream of the $\mathrm{H} 37 \mathrm{Rv}$ annotated start codon. Six possible start codons are underlined; the annotated start site of $M$. tuberculosis strain CDC1551 is indicated by $\dagger$, that of H37Rv by $\neq$. To determine the translational start codon, single-residue deletions (bold and numbered) were created between potential start codons. (b) Western blot analysis of cell-free extracts of $M$. smegmatis containing pEJMyc, pKSO3, pKSO3mut2, pKS03mut3, pKS03mut4, pKS03mut5 or pKS03mut6. A $21.6 \mathrm{kDa}$ band corresponding to the smallest SigC-Myc can be detected from strains containing pKS03, pKS03mut2, pKS03mut3, pKS03mut4 and pKS03mut5 but not pEJMyc or pKS03mut6. No larger SigC-Myc of $35.4 \mathrm{kDa}$ can be detected in any strain. (c) RT-PCR analysis of RNA extracts of M. smegmatis containing pEJMyc, pKS03 or pKS03mut6. A 225 bp band indicating the presence of sigC-myc transcript was detected from strains containing pKS03 and pKS03mut6 but not strains containing pEJMyc or the RNA without RT controls. (d) Western blot analysis of cellfree extracts of $M$. tuberculosis containing pEJMyc, pKS03, pKS03mut5 or pKS03mut6. (e) RT-PCR analysis of RNA extracts of $M$. tuberculosis containing pEJMyc, pKS03, or pKS03mut6. (f) Western blot analysis of cell-free extracts of $M$. tuberculosis containing pEJMyc, pJS014 or pKS03 at different stages of growth indicated by $\mathrm{OD}_{600}$. A $21.6 \mathrm{kDa}$ band corresponding to SigC-Myc can be detected from strains containing pKS03 at all growth phases. No larger SigC-Myc of $35.4 \mathrm{kDa}$ with pKS03 or $10.5 \mathrm{kDa}$ truncated SigC-Myc with pJS014 can be detected at any growth stage examined. (g) RT-PCR analysis of RNA extracts of M. tuberculosis containing pEJMyc or pJS014 at different growth stages. 
(a)

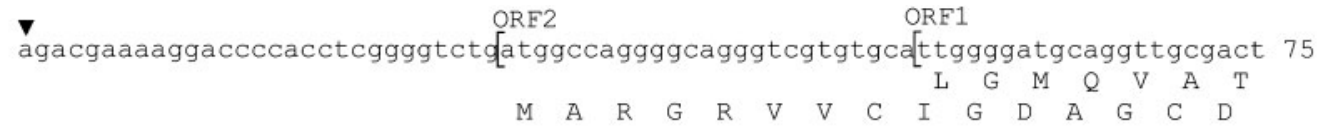

gtacacccggcgtgttccgcgcgacagcgggtgggatgccggtgctggtggtcatcgagtctgggacaggaggtg 150

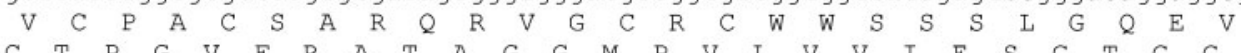

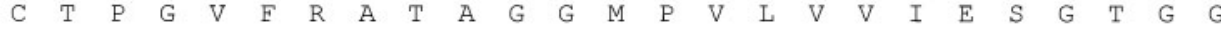

atcagatggctcgtaaagctacgtccccgggtaagccggctccgacgtcgggacagtatcgcccggttggcggtg 225 $\begin{array}{llllllllllllllllllllllllllll}I & R & W & L & V & K & L & R & P & R & V & S & R & L & R & R & R & D & S & I & A & R & L & A & V\end{array}$

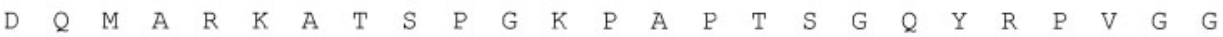
$\mathrm{ORF} 1$

gcaacgaggtgajccgttccgaagggacaccgtctgcctccctcgcccaagcccggtcagaagtgggtgaacgtcg 300 A $T$ R *

$\begin{array}{lllllllllllllllllllllllllllllllllll}G & N & E & V & T & V & P & K & G & H & R & L & P & P & S & P & K & P & G & Q & K & W & V & N & V\end{array}$

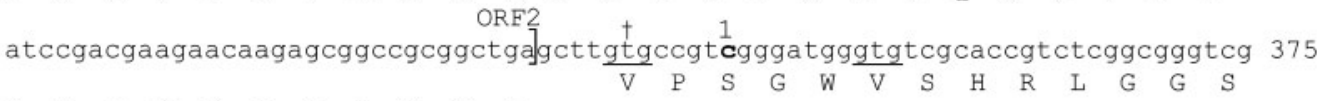
$\begin{array}{llllllllllllllllllllll}D & P & \text { T } & K & N & L & S & G & R & G & *\end{array}$

cccaagtgcataagtgctttgtcgetgccetccggtaccgtcggagccccgtccaagccggacaacgacgccact 450 $\begin{array}{llllllllllllllllllllllllllllllll}P & K & C & I & S & A & L & S & L & P & S & G & T & A & G & A & P & S & K & P & D & N & D & A & \text { T }\end{array}$

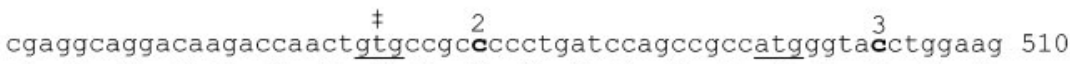

(b)

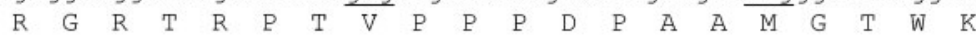

Mitc
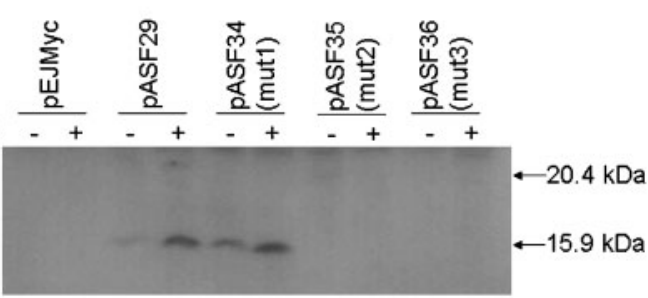

(d)

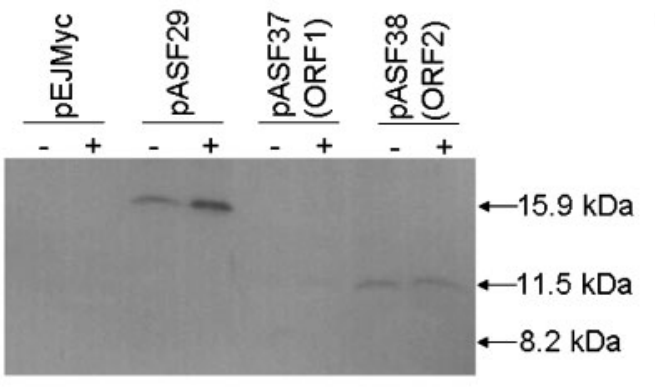

(c)

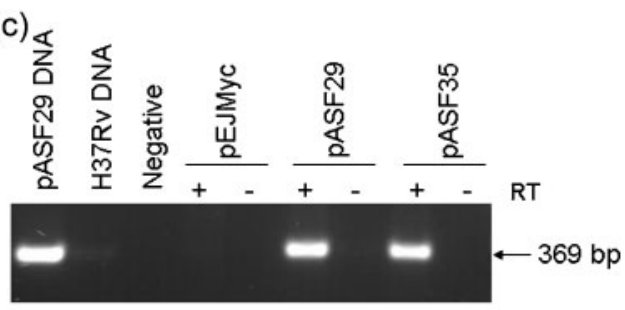

(e)
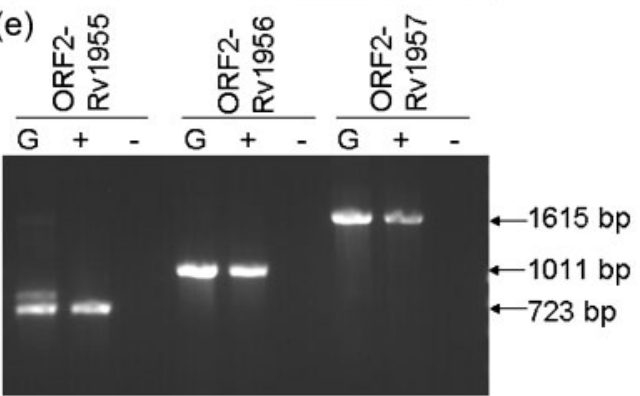

Fig. 4. Identification of the transcriptional and translational start sites of Rv1955 and the discovery of a novel ORF upstream of Rv1955. (a) The nucleotide sequence of the beginning of the Rv1955 gene including 336 bp upstream of the annotated start codon. The RecA-NDp motif is shaded and the transcriptional start sites identified by $5^{\prime}$ RACE are shown $(\boldsymbol{\nabla})$. Possible start codons are underlined; the annotated start site is indicated by $\dagger$ and the experimentally defined start codon is indicated by $\ddagger$. To determine the translational start codon, single-residue deletions (bold and numbered) were created between potential start codons. The location of two potential ORFs upstream of Rv1955 are indicated with square brackets, and their amino acid translations are given. (b) Western blot analysis of cell-free extracts of $M$. tuberculosis containing pEJMyc, pASF29, pASF34 (containing mutation 1), pASF35 (mutation 2) or pASF36 (mutation 3), from cultures with and without the addition of mitomycin C (MitC). A $15.9 \mathrm{kDa}$ band corresponding to Rv1955-Myc can be detected from strains containing pASF29 and pASF34 but not pEJMyc, pASF35 or pASF36. The effect of mitomycin C-induced DNA damage on expression levels of Rv1955-Myc can be seen. (c) RT-PCR analysis of RNA extracts of $M$. tuberculosis containing pEJMyc, pASF29 or pASF35. A 369 bp band indicating the presence of Rv1955-myc transcript was detected from strains containing pASF29 and pASF35 but not from strains containing pEJMyc or the RNA without RT controls. (d) Western blot analysis of cell-free extracts of $M$. tuberculosis containing pEJMyc, pASF29, pASF37 (ORF1) or pASF38 (ORF2), from cultures with and without the addition of mitomycin C. Again a $15.9 \mathrm{kDa}$ band corresponding to Rv1955-Myc can be detected from the strain containing pASF29. A potential 8.2 kDa ORF1-Myc protein was undetectable from pASF37. A $11.5 \mathrm{kDa}$ band corresponding to ORF2-Myc can be detected from the strain containing pASF38 but not from strains containing pASF28, pASF37 or pEJmyc. (e) RT-PCR analysis of an $M$. tuberculosis H37Rv RNA extract shows that ORF2 is co-transcribed with Rv1955-Rv1957. The $723 \mathrm{bp}, 1011 \mathrm{bp}$ and 1615 bp bands indicate the presence of ORF2-Rv1955, ORF2-Rv1956 and ORF2-Rv1957 transcripts within cDNA from $M$. tuberculosis H37Rv (G, genomic DNA positive control; +, RT positive cDNA; -, RT negative control). 
pASF36, where residues 1-3 were deleted respectively (Fig. 4a). Rv1955-Myc was undetectable from all extracts of M. smegmatis $\mathrm{mc}^{2} 155$ containing pASF29 or its derivatives (data not shown). The plasmids were therefore transformed into M. tuberculosis $\mathrm{H} 37 \mathrm{Rv}$ and, in an attempt to increase Rv1955-Myc expression levels, cell-free extract was prepared from cultures with mitomycin C-induced DNA damage alongside uninduced control cultures. Upon Western blotting, Myc-tagged Rv1955 was expected to be detected between $20.4 \mathrm{kDa}$ and $15.1 \mathrm{kDa}$, for the largest and smallest potential ORFs, respectively. Rv1955-Myc was detected in extracts from uninduced cultures, and to a greater extent in induced cultures, from $M$. tuberculosis containing pASF29 and pASF34 at $15.9 \mathrm{kDa}$, but was not detected in either extract from $M$. tuberculosis containing pEJMyc, pASF35 or pASF36 (Fig. 4b). The presence of mRNA transcript for Rv1955-myc was confirmed by RTPCR using primers Rv1955RTF and MycRTR, indicating that the mutations introduced had no effect on transcription (Fig. 4c). This shows that the translational start site for Rv1955 is between residues 1 and 2, indicating that Rv1955 is not translated from the annotated start site. Furthermore, the translational start cannot be upstream of the transcription start site, so translation must begin from the GTG start codon $135 \mathrm{bp}$ downstream of that annotated. The increased expression level of Rv1955-Myc upon the addition of mitomycin $\mathrm{C}$ supports the observation that the predicted DNA damage inducible promoter RecA-NDp controls Rv1955-Rv1957 expression.

\section{Identification of a novel ORF upstream of Rv1955}

As the Rv1955 translational start site was determined to be $135 \mathrm{bp}$ downstream of the annotated start site, there is a $471 \mathrm{bp}$ region between the furthest upstream transcriptional start site and the Rv1955 translational start site. This $471 \mathrm{bp}$ region was therefore analysed for the presence of any potential ORFs. Two possible ORFs were identified and pASF29 was mutated to bring these potential ORFs into frame with the Myc tag, to give pASF37 and pASF38 for ORF1 and ORF2 respectively (Fig. 4a). pASF37 and pASF38 were transformed into $M$. tuberculosis $\mathrm{H} 37 \mathrm{Rv}$ and cell-free extract was prepared from cultures with and without mitomycin C-induced DNA damage. Upon Western blotting, ORF1-Myc and ORF2-Myc were expected to be detected at a maximum of $8.2 \mathrm{kDa}$ and $11.5 \mathrm{kDa}$ respectively. ORF1-Myc was undetectable in both extracts of $M$. tuberculosis containing pASF37 (Fig. 4d). However, ORF2Myc was detected in extracts from the uninduced culture, and to a similar extent in the induced culture, from $M$. tuberculosis containing pASF38, but was not detected in either extract from M. tuberculosis containing pEJMyc (Fig. 4d). We have therefore demonstrated that a small, non-annotated ORF upstream of Rv1955 is expressed as a protein within M. tuberculosis $\mathrm{H} 37 \mathrm{Rv}$.

Searching the protein database revealed no conserved domains to indicate a possible function for ORF2, nor any significant sequence homology to other bacterial proteins using BLASTP (Altschul et al., 1990). The expression level of ORF2-Myc does not appear to increase upon the addition of mitomycin $\mathrm{C}$, suggesting that the $\mathrm{P} 2$ promoter is not DNA damage inducible. To determine whether ORF2 is cotranscribed with Rv1955-Rv1957, RT-PCR was performed on cDNA generated from $M$. tuberculosis $\mathrm{H} 37 \mathrm{Rv}$ RNA using primers spanning the region from ORF2 to Rv1955, Rv1956 and Rv1957 individually. Specific transcripts were detected using all three primer pairs, indicating that ORF2 is part of the Rv1955-Rv1957 operon (Fig. 4e).

\section{DISCUSSION}

The annotation of bacterial genomes is performed using mathematical models, often with comparison to other genomes and protein databases. As the available information increases these predictions become more accurate; however, not all ORF predictions can be assumed to be correct. This is particularly true of the translational start site, which tends to be more difficult to predict. The initial annotation of ORFs in the M. tuberculosis H37Rv genome identified 3974 potential genes (Cole et al., 1998). Reannotation 4 years later led to the identification of 82 new potential genes and the lengths of 60 ORFs being altered, the majority of which were altered at the Nterminus (Camus et al., 2002). We suspected that the translational start sites for a number of $M$. tuberculosis $\mathrm{H} 37 \mathrm{Rv}$ genes remain incorrectly annotated, and so we devised a simple approach for determining translational start sites using a combination of epitope tagging and frameshift mutagenesis. Using this assay we were able to determine the translational start sites of three proteins. Of these, LexA was shown to start upstream of the annotated start site, the H37Rv annotated start site was confirmed for SigC, and Rv1955 was shown to start downstream of the annotated start site. We were also able to identify that a previously unrecognized ORF upstream of Rv1955 is expressed as a protein.

The negative regulator LexA is one of the key players in the SOS response, a bacterial response to DNA damage, and homologues have been characterized from both $M$. tuberculosis and M. smegmatis (Davis et al., 2002; Durbach et al., 1997; Movahedzadeh et al., 1997). LexA represses transcription of DNA damage inducible genes by binding to an upstream DNA sequence termed the SOS box. DNA damage activates RecA, which in turn stimulates autocatalytic cleavage of LexA, lifting repression of the regulated genes. The initial annotation of the M. tuberculosis LexA identified the translational start site based on comparison to the identified LexA sequence from other bacterial species (Movahedzadeh et al., 1997). Since then a number of mycobacterial genomes have been sequenced, the annotation of which in many cases shows a longer $\mathrm{N}$ terminal sequence for LexA, leading us to re-evaluate the M. tuberculosis annotation. We were able to demonstrate that the translational start site of LexA is 57 bases upstream 
of the annotated start, at the same location as the transcriptional start site. Such leaderless mRNAs, with no $5^{\prime}$ untranslated regions, are an increasingly recognized phenomenon and are particularly prevalent in Grampositive bacteria and archaea (Moll et al., 2002). This alternative translational start site is also likely to apply in $M$. bovis as, although it is annotated to start at a similar site to LexA of E. coli and B. subtilis (Fig. 1), the N-terminal nucleotide sequence is identical to that of $M$. tuberculosis (data not shown).

Bacterial sigma factors are components of RNA polymerase that bind to specific DNA promoter regions, thus directing the transcription of specific genes. Sigma factor C (SigC) is an extracytoplasmic function sigma factor; this group of sigma factors is involved in the response to stress conditions (Rodrigue et al., 2006). Although the stimulus for SigC activation is not known it has been predicted to be involved in virulence as it is present in the genomes of $M$. tuberculosis and $M$. leprae but absent from the nonpathogenic strain M. smegmatis (Cole et al., 1998, 2001; Waagmeester et al., 2005). Indeed, SigC has been shown to play a role in pathogenesis in mouse and guinea pig models of M. tuberculosis infections (Karls et al., 2006; Sun et al., 2004). The annotation for sigC in two M. tuberculosis strains differed significantly in the $5^{\prime}$ region, with sigC from strain CDC1551 starting 378 nucleotides further upstream than the gene from strain H37Rv (Camus et al., 2002; Fleischmann et al., 2002). We used our assay to confirm that the annotated start site for H37Rv was correct for this strain. It is likely that the H37Rv annotation is also correct for strain CDC1551, as the sequences over this region are identical. Furthermore, the translational apparatus is highly conserved in these two strains, with the sequences of the majority of ribosomal and initiation factor genes being identical, making translation from an alternative site unlikely. This contradicts a recent investigation, in which the size of SigC present in cell-free extracts from $\mathrm{H} 37 \mathrm{Rv}$ was compared to recombinant protein by Western blotting, leading to the conclusion that the CDC1551 annotation was correct (Thakur et al., 2007). We therefore investigated the possibility that translation initiates from different sites under different growth conditions. Such overlapping genes are well documented in eukaryotes and viruses, and have also been identified in several species of bacteria including E. coli, Bacillus subtilis and Corynebacterium flavum (Chen \& Paulus, 1988; Follettie et al., 1993; Normark et al., 1983; Plumbridge et al., 1985). However, we were unable to find any evidence for alternative translation initiation in the sigC gene in this investigation.

The predicted transcriptional regulator Rv1956 forms an operon with two genes of unknown function, Rv1955 and Rv1957 (Cole et al., 1998; Rand, 2003). Gene expression analyses indicate that the Rv1955-Rv1957 operon is upregulated when $M$. tuberculosis is exposed to heat shock (Stewart et al., 2002), nutrient starvation (Betts et al., 2002) and DNA damage (Rand et al., 2003). The association of the RecA-NDp promoter motif with Rv1955 suggests that this operon may form part of the LexA/RecA-independent response to DNA damage (Gamulin et al., 2004). As the RecA-NDp promoter was found to be within the annotated translated region of Rv1955 we determined the transcriptional and translational start sites of Rv1955 experimentally. Two transcriptional start sites were identified for Rv1955, one downstream and the other upstream of the annotated translation start site, suggesting that two promoters (designated $\mathrm{P} 1$ and $\mathrm{P} 2$ respectively) control the expression of the Rv1955-Rv1957 operon. The translational start site was found to be $135 \mathrm{bp}$ downstream of the annotated start site and is downstream of both the RecA-NDp motif and the two identified transcriptional start sites. This, along with the observation that Rv1955 is more highly expressed after mitomycin C induction, supports the notion that this operon may form part of the LexA/RecA-independent DNA damage response.

In this investigation we were also able to use the C-terminal Myc tag to identify that a novel ORF located upstream of Rv1955 is expressed as a protein. We suggest that this new ORF be renamed Rv1954A, in keeping with current naming consensus (Camus et al., 2002). Rv1954A does not contain any conserved protein domains and its function remains unknown. Small proteins often remain unannotated within genome annotations as the methods used often exclude potential ORFs below a certain length. The initial $M$. tuberculosis $\mathrm{H} 37 \mathrm{Rv}$ annotation limited ORF length to 100 aa (Cole et al., 1998) and reannotation reduced this to 60 aa, resulting in the identification of many more potential ORFs (Camus et al., 2002). Rv1954A is 100 aa in length but was not identified in either annotation. This is probably because Rv1954A overlaps with Rv1954c, which is located on the opposite strand (Cole et al., 1998). However there is no experimental evidence that Rv1954c is expressed as a protein, and this gene is not annotated in the M. tuberculosis strain CDC1551 genome (Fleischmann et al., 2002), so it may represent a mis-annotation in H37Rv.

The Rv1954A gene lies between the two identified transcriptional start sites of Rv1955. Rv1954A is therefore only expressed from the $\mathrm{P} 2$ promoter, which, unlike the $\mathrm{P} 1$ promoter, does not appear to be DNA damage inducible. After RT-PCR analysis it appears that Rv1954A-Rv1957 form a single operon and that the $\mathrm{P} 2$ promoter controls the expression of all these proteins under the growth conditions used in the study, and perhaps upon the addition of an unknown stimulus. The P1 promoter also appears to control the expression of Rv1955-Rv1957 upon DNA damage.

In conclusion, we have devised a simple method for determining the translational start sites of predicted ORFs and demonstrated its use on a number of mycobacterial proteins. We have also used this method to identify a previously unannotated ORF, Rv1954A. For many ORFs, but not all, it is possible to perform this assay in $M$. smegmatis, a model organism for M. tuberculosis, which 
grows faster than M. tuberculosis and is easier to work with as it is an ACDP containment level 1 organism. This may be the case even if the gene is not present in the $M$. smegmatis genome, as homologues of many of the regulatory factors required for the translation of $M$. tuberculosis genes are conserved between M. tuberculosis and M. smegmatis (Tyagi \& Sharma, 2002). For example, we were able to detect SigC-Myc even though no native $\mathrm{SigC}$ is present in the M. smegmatis genome (Waagmeester et al., 2005). It is likely that the translation initiation sites and new ORFs outlined in this paper also apply to other members of the M. tuberculosis complex.

\section{ACKNOWLEDGEMENTS}

This study was funded by the Medical Research Council.

\section{REFERENCES}

Altschul, S. F., Gish, W., Miller, W., Myers, E. W. \& Lipman, D. J. (1990). Basic local alignment search tool. J Mol Biol 215, 403-410.

Besemer, J., Lomsadze, A. \& Borodovsky, M. (2001). GeneMarkS: a self-training method for prediction of gene starts in microbial genomes. Implications for finding sequence motifs in regulatory regions. Nucleic Acids Res 29, 2607-2618.

Betts, J. C., Lukey, P. T., Robb, L. C., McAdam, R. A. \& Duncan, K. (2002). Evaluation of a nutrient starvation model of Mycobacterium tuberculosis persistence by gene and protein expression profiling. Mol Microbiol 43, 717-731.

Brosch, R., Gordon, S. V., Garnier, T., Eiglmeier, K., Frigui, W., Valenti, P., Dos Santos, S., Duthoy, S., Lacroix, C. \& other authors (2007). Genome plasticity of BCG and impact on vaccine efficacy. Proc Natl Acad Sci U S A 104, 5596-5601.

Camus, J. C., Pryor, M. J., Medigue, C. \& Cole, S. T. (2002). Reannotation of the genome sequence of Mycobacterium tuberculosis H37Rv. Microbiology 148, 2967-2973.

Carter, R. J., Dubchak, I. \& Holbrook, S. R. (2001). A computational approach to identify genes for functional RNAs in genomic sequences. Nucleic Acids Res 29, 3928-3938.

Chen, N. Y. \& Paulus, H. (1988). Mechanism of expression of the overlapping genes of Bacillus subtilis aspartokinase II. J Biol Chem 263, 9526-9532.

Cole, S. T., Brosch, R., Parkhill, J., Garnier, T., Churcher, C., Harris, D., Gordon, S. V., Eiglmeier, K., Gas, S. \& other authors (1998). Deciphering the biology of Mycobacterium tuberculosis from the complete genome sequence. Nature 393, 537-544.

Cole, S. T., Eiglmeier, K., Parkhill, J., James, K. D., Thomson, N. R., Wheeler, P. R., Honore, N., Garnier, T., Churcher, C. \& other authors (2001). Massive gene decay in the leprosy bacillus. Nature 409, 1007-1011.

Davis, E. O., Dullaghan, E. M. \& Rand, L. (2002). Definition of the mycobacterial SOS box and use to identify LexA-regulated genes in Mycobacterium tuberculosis. J Bacteriol 184, 3287-3295.

Delcher, A. L., Harmon, D., Kasif, S., White, O. \& Salzberg, S. L. (1999). Improved microbial gene identification with GLIMMER. Nucleic Acids Res 27, 4636-4641.

Durbach, S. I., Andersen, S. J. \& Mizrahi, V. (1997). SOS induction in mycobacteria: analysis of the DNA-binding activity of a LexA-like repressor and its role in DNA damage induction of the recA gene from Mycobacterium smegmatis. Mol Microbiol 26, 643-653.
Edman, P. J. (1950). Method for determination of the amino acid sequence in peptides. Acta Chem Scand 4, 283-293.

Fleischmann, R. D., Alland, D., Eisen, J. A., Carpenter, L., White, O., Peterson, J., DeBoy, R., Dodson, R., Gwinn, M. \& other authors (2002). Whole-genome comparison of Mycobacterium tuberculosis clinical and laboratory strains. J Bacteriol 184, 5479-5490.

Follettie, M. T., Peoples, O. P., Agoropoulou, C. \& Sinskey, A. J. (1993). Gene structure and expression of the Corynebacterium flavum N13 ask-asd operon. J Bacteriol 175, 4096-4103.

Gamulin, V., Cetkovic, H. \& Ahel, I. (2004). Identification of a promoter motif regulating the major DNA damage response mechanism of Mycobacterium tuberculosis. FEMS Microbiol Lett 238, 57-63.

Karls, R. K., Guarner, J., McMurray, D. N., Birkness, K. A. \& Quinn, F. D. (2006). Examination of Mycobacterium tuberculosis sigma factor mutants using low-dose aerosol infection of guinea pigs suggests a role for SigC in pathogenesis. Microbiology 152, 1591-1600.

Makita, Y., de Hoon, M. \& Danchin, A. (2007). Hon-yaku: a biologydriven Bayesian methodology for identifying translation initiation sites in prokaryotes. BMC Bioinformatics 8, 47.

Moll, I., Grill, S., Gualerzi, C. O. \& Blasi, U. (2002). Leaderless mRNAs in bacteria: surprises in ribosomal recruitment and translational control. Mol Microbiol 43, 239-246.

Moreno-Hagelsieb, G. \& Collado-Vides, J. (2002). A powerful nonhomology method for the prediction of operons in prokaryotes. Bioinformatics 18, S329-S336.

Movahedzadeh, F., Colston, M. J. \& Davis, E. O. (1997). Characterization of Mycobacterium tuberculosis LexA: recognition of a Cheo (Bacillus-type SOS) box. Microbiology 143, 929-936.

Nielsen, P. \& Krogh, A. (2005). Large-scale prokaryotic gene prediction and comparison to genome annotation. Bioinformatics 21, 4322-4329.

Normark, S., Bergstrom, S., Edlund, T., Grundstrom, T., Jaurin, B., Lindberg, F. P. \& Olsson, O. (1983). Overlapping genes. Annu Rev Genet 17, 499-525.

Plumbridge, J. A., Deville, F., Sacerdot, C., Petersen, H. U., Cenatiempo, Y., Cozzone, A., Grunberg-Manago, M. \& Hershey, J. W. (1985). Two translational initiation sites in the infB gene are used to express initiation factor IF2 alpha and IF2 beta in Escherichia coli. EMBO J 4, 223-229.

Rand, L. (2003).The role of DNA repair genes in Mycobacterium tuberculosis pathogenesis. $\mathrm{PhD}$ thesis. University College London.

Rand, L., Hinds, J., Springer, B., Sander, P., Buxton, R. S. \& Davis, E. O. (2003). The majority of inducible DNA repair genes in Mycobacterium tuberculosis are induced independently of RecA. Mol Microbiol 50, 1031-1042.

Rison, S. C. G., Mattow, J., Jungblut, P. R. \& Stoker, N. G. (2007). Experimental determination of translational starts using peptide mass mapping and tandem mass spectrometry within the proteome of Mycobacterium tuberculosis. Microbiology 153, 521-528.

Rodrigue, S., Provvedi, R., Jacques, P. E., Gaudreau, L. \& Manganelli, R. (2006). The sigma factors of Mycobacterium tuberculosis. FEMS Microbiol Rev 30, 926-941.

Sambrook, J., Fritsch, E. \& Maniatis, T. (1989). Molecular Cloning: $a$ Laboratory Manual. Cold Spring Harbor, NY: Cold Spring Harbor Laboratory.

Snapper, S. B., Melton, R. E., Mustafa, S., Kieser, T. \& Jacobs, W. R., Jr (1990). Isolation and characterization of efficient plasmid transformation mutants of Mycobacterium smegmatis. Mol Microbiol 4, 1911-1919. 
Spratt, J. M., Ryan, A. A., Britton, W. J. \& Triccas, J. A. (2005). Epitopetagging vectors for the expression and detection of recombinant proteins in mycobacteria. Plasmid 53, 269-273.

Stewart, G. R., Wernisch, L., Stabler, R., Mangan, J. A., Hinds, J., Laing, K. G., Young, D. B. \& Butcher, P. D. (2002). Dissection of the heat-shock response in Mycobacterium tuberculosis using mutants and microarrays. Microbiology 148, 3129-3138.

Stinear, T. P., Seemann, T., Pidot, S., Frigui, W., Reysset, G., Garnier, T., Meurice, G., Simon, D., Bouchier, C. \& other authors (2007). Reductive evolution and niche adaptation inferred from the genome of Mycobacterium ulcerans, the causative agent of Buruli ulcer. Genome Res 17, 192-200.

Stover, C. K., de la Cruz, V. F., Fuerst, T. R., Burlein, J. E., Benson, L. A., Bennett, L. T., Bansal, G. P., Young, J. F., Lee, M. H. \& other authors (1991). New use of BCG for recombinant vaccines. Nature 351, 456-460.
Sun, R., Converse, P. J., Ko, C., Tyagi, S., Morrison, N. E. \& Bishai, W. R. (2004). Mycobacterium tuberculosis ECF sigma factor sigC is required for lethality in mice and for the conditional expression of a defined gene set. Mol Microbiol 52, 25-38.

Thakur, K. G., Joshi, A. M. \& Gopal, B. (2007). Structural and biophysical studies on two promoter recognition domains of the extra-cytoplasmic function $\sigma$ factor $\sigma^{\mathrm{C}}$ from Mycobacterium tuberculosis. J Biol Chem 282, 4711-4718.

Tyagi, J. S. \& Sharma, D. (2002). Mycobacterium smegmatis and tuberculosis. Trends Microbiol 10, 68-69.

Waagmeester, A., Thompson, J. \& Reyrat, J.-M. (2005). Identifying sigma factors in Mycobacterium smegmatis by comparative genomic analysis. Trends Microbiol 13, 505-509.

Edited by: D. W. Ussery 